\title{
Simulation model of wax diffusion and cleaning in printer belts
}

\author{
S. J. L. van Eijndhoven · D. P. Siregar • T. Siebers
}

Received: 19 April 2010 / Accepted: 6 May 2011 / Published online: 8 June 2011

(C) The Author(s) 2011. This article is published with open access at Springerlink.com

\begin{abstract}
A belt that transports toner is one of the vital components of a printer. Since toner is fused to the paper at a high temperature, wax releases from the paper and penetrates into the rubber top layer of the belt. When the rubber becomes saturated with wax, the wax remains on top of the belt. The formed layer of wax has negative impact on the image forming unit leading to bad printing quality. Thus, a wax cleaner is installed. To determine optimal functioning of the cleaner, time consuming and inefficient experiments have to be carried out. Thus, an efficient simulation tool to predict wax build-up and cleaning may replace the experiments. Simulation is based on a mathematical model that describes the influx of wax as a convection/diffusion process. The standard numerical discretization methods to calculate the evolution in time of the wax concentration are not applicable. Saturation is reached after ten thousands of rounds. In this article, we propose a combination of an analytical and a numerical method to tackle the problem, where we discretize the second-order differential operator that generates the evolution of the wax concentration. The simulations show an adequate fit with results from measurement. The wax build-up in the belt up to saturation is described realistically. Our study reveals that the contact resistance between belt and cleaner is the most important parameter that influences the effectiveness of the cleaner.
\end{abstract}

Keywords (Wax) Diffusion · Eigenvalue $\cdot$ Multi-layered medium $\cdot$ Printing belt $\cdot$ Printing system

\section{Introduction}

In the design of a printer, the life-span of the belt that transports the toner is a main aspect. The main use of this belt is to transfer the toner image from the image forming unit to the fuse nip, where the toner is fixed to the paper. At the fuse nip the paper is heated up and wax contained in the paper flows out of the paper and penetrates the rubber top

S. J. L. van Eijndhoven

Department of Mathematics and Computer Science, Eindhoven University of Technology, 5600 MB Eindhoven, The Netherlands

e-mail: s.j.1.v.eijndhoven@tue.nl

D. P. Siregar $(\varangle)$

Department of Mechanical Engineering, Eindhoven University of Technology, 5600 MB Eindhoven, The Netherlands

e-mail: d.p.siregar@tue.nl

T. Siebers

Océ Technologies B.V, 5914 CA Venlo, The Netherlands 


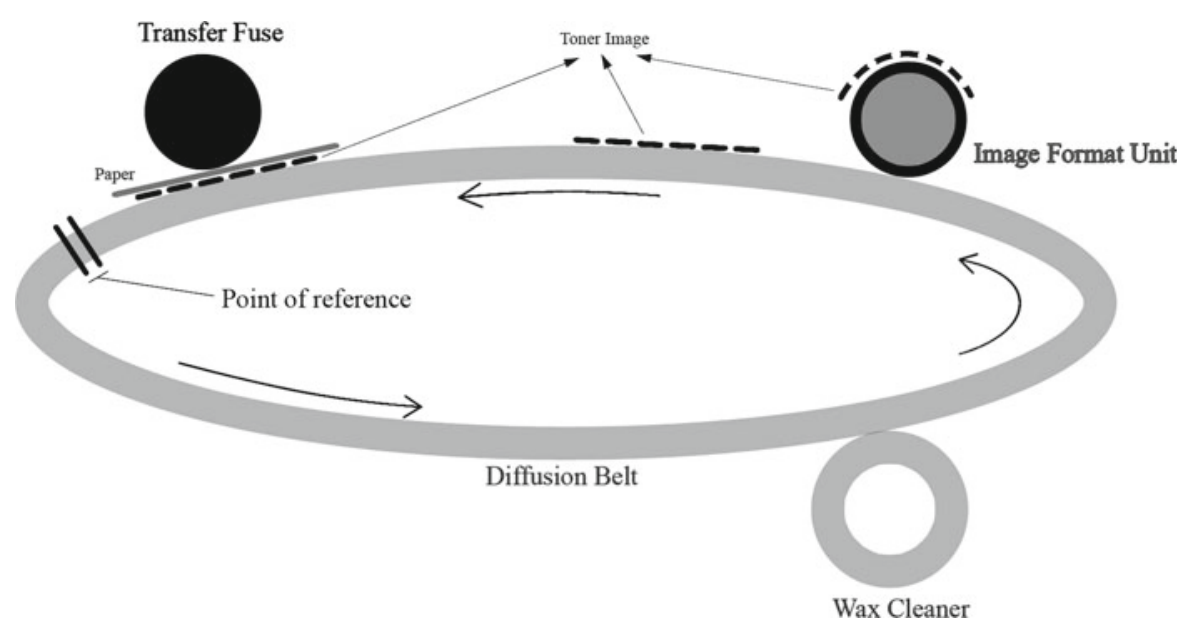

Fig. 1 The schematic of the system. Here, we indicate the point of reference for the modeling

layer of the belt. Until saturation is reached, wax is absorbed by the belt. At saturation, the belt is no longer capable of absorbing wax and a thin layer of wax forms at the top of the belt. This wax layer pollutes components attached to the belt and thus reduces the printing quality significantly. Consequently, the belt has to be replaced, which is a costly procedure. In order to prolong the belt's life-span, a wax cleaner is installed. The wax cleaner decomposes part of the wax and slows down the wax pollution. For every 20,000 copies that the printer makes, the wax in the cleaner is removed by lifting up the cleaner, increasing its temperature to $140^{\circ} \mathrm{C}$ for approximately $100 \mathrm{~min}$. If the wax is removed, the cleaner is positioned again in the system automatically. During the removal of the wax from the cleaner the printing process may keep on running (Fig. 1).

In this article, we describe a mathematical model for wax diffusion and cleaning including saturation effects. The wax diffuses into a multi-layered belt due to wax influx at a position corresponding to the fuse nip location and is partly removed at the position corresponding to the location of the cleaner due to contact between belt and cleaner. In the model, we incorporate the cyclic motion of the belt, diffusion in transversal direction, and diffusion into the cleaner that decomposes the wax. We are interested in the influence of certain physical parameters on the process, such as the inverse contact resistance between belt and cleaner, the inverse contact resistances between sublayers of the belt, the decomposition rate of the cleaner, and various diffusion constants.

The large number of belt rotations and the small amounts of wax diffusing into the belt make time discretization inappropriate. Thus, a dedicated implementation of the model is required. We propose a combination of analytical and numerical techniques, where the time evolution is described by a finite number of eigenfunctions and eigenvalues of a second-order differential operator. Although the real life problem in this paper is typical, there are generic aspects covered that can also be found in literature. In this, we refer to the papers of Wang and Liu [1], de Monte [2], Pontrelli and de Monte [3], Tamene et al. [4], and Hickson et al. [5] that address heat conduction in composite media. We also refer to the previous works of Schuurmans [10], Kopinga [11], Zavinska [12], and Zapata [13] that address a similar problem.

We validated our model with respect to data obtained from measurement. ${ }^{1}$ Computation time is acceptable (12,500 belt rotations need approximately $5 \mathrm{~min}$ ), but can be improved if the Matlab implementation is replaced by a professional platform independent implementation.

This article is composed of six sections. In Sect. 2, we present the mathematical model, describing both diffusion of wax in moving belt and cleaner. The solution procedure is outlined in Sects. 3 and 4 contains the details of numerical implementation. In Sect. 5, numerical results are presented, validated with experimental data obtained from the measurement in the company. Section 6 concludes.

\footnotetext{
1 The authors acknowledge cooperation with Océ Technologies B.V. and the use of measurement.
} 


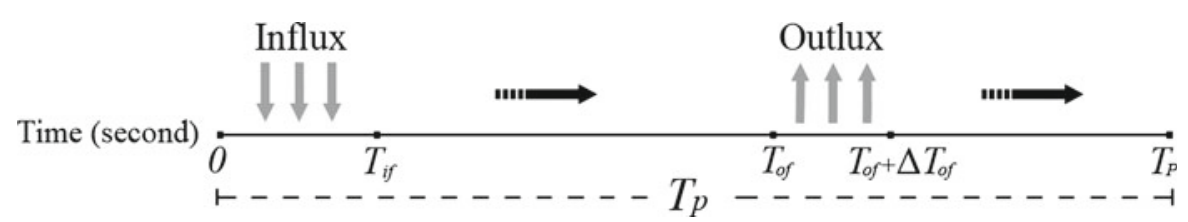

Fig. 2 The time frame for the first cycle of the process in the belt. Time is indicated along the horizontal axis to describe the processes in certain time intervals

\section{Mathematical model}

A round belt with length $L$ moves with a constant speed $V$. The wax concentration is determined by a wax influx at the fuse nip and a wax outflux at the cleaner. The reference frame is chosen such that $\mathbf{e}_{x}$ is the direction of the motion of the belt; thus the belt velocity is given by $\mathbf{v}=V \mathbf{e}_{x}, \mathbf{e}_{y}$ is in the transversal direction and $\mathbf{e}_{z}$ is in the normal to the plane of motion.

The assumption is that in $z$-direction we have uniform influx and outflux so that the wax concentration in the belt is independent of $z$. Moreover, the wax transport by diffusion is on a much lower time scale than the wax transport by convection due to the motion of the belt, i.e., $L^{2} / \kappa \gg L / V$, where $\kappa$ denotes the diffusivity. Thus, the problem can be described as a one-dimensional diffusion problem. The wax concentration of the belt is given by

$C_{\mathrm{b}}(x, y, z, t)=C_{\mathrm{b}}\left(y, t-\frac{x}{V}\right)$

The wax influx at the fuse nip position is uniform and time independent; the wax outflux from the belt to cleaner is linearly related to the difference of wax concentrations of belt and cleaner at contact area.

For one rotation of the belt, which has a duration of $T_{\mathrm{p}}=L / V$, the time scheme of the reference position at the belt is depicted in Fig. 2. The length of the fuse nip, $L_{\text {if }}$, is described by the time parameter $T_{\text {if }}$ according to $T_{\text {if }}=L_{\text {if }} / V$, so in a time interval of length $T_{\text {if }}$ wax flows into the belt. As starting time of any revolution we take the moment the belt reference position enters the fuse nip. Then, the reference position enters the cleaning zone at time $T_{\text {of }}$ and stays in the cleaning zone for a duration of time $\Delta T_{\text {of }}$ so that it leaves the cleaning zone at time $T_{\text {of }}+\Delta T_{\text {of }}$.

The process starts at $t=0$. There is first wax influx from paper to belt in the interval $0<t<T_{\text {if }}$. Wax outflux to the cleaner takes place in the time interval $T_{\text {of }}<t<T_{\text {of }}+\Delta T_{\text {of }}$. At the start of the $n$th round, the $n$th wax influx from paper to belt takes place in the time interval $(n-1) T_{\mathrm{p}}<t<(n-1) T_{\mathrm{p}}+T_{\text {if }}$ and wax outflux in the time interval $(n-1) T_{\mathrm{p}}+T_{\text {of }}<t<(n-1) T_{\mathrm{p}}+T_{\text {of }}+\Delta T_{\text {of }}$.

The belt consists of two physical layers, a top layer and a bottom layer; see Fig. 3. The top thin layer is described by $y_{0}<y<y_{1}$, where the contact with the paper and the cleaner takes place at $y=y_{0}$. The bottom layer is described by the interval $y_{1}<y<y_{2}$; at $y=y_{2}$ we assume no outflux of wax. The two layers are composed of different material with diffusivities $\kappa_{1}$ and $\kappa_{2}$. At the contact $y=y_{1}$, the wax flux is continuous; it is assumed proportional to the difference of wax concentrations at the contact with a proportionality factor called inverse resistance. Thus, for the wax concentration in the belt, we have the following model:

$\frac{\partial C_{\mathrm{b}}}{\partial t}=\kappa_{i} \frac{\partial^{2} C_{\mathrm{b}}}{\partial y^{2}}, \quad y_{i-1}<y<y_{i}, \quad t>0, \quad i=1,2$.

The boundary and interface conditions are given by

$$
\begin{aligned}
\kappa_{1}\left[\frac{\partial C_{\mathrm{b}}}{\partial y}\right]_{y=y_{0}} & =\Theta(t) \\
\kappa_{1}\left[\frac{\partial C_{\mathrm{b}}}{\partial y}\right]_{y=y_{1}^{-}} & =\kappa_{2}\left[\frac{\partial C_{\mathrm{b}}}{\partial y}\right]_{y=y_{1}^{+}} \\
\kappa_{1}\left[\frac{\partial C_{\mathrm{b}}}{\partial y}\right]_{y=y_{1}^{-}} & =h_{12}\left(C_{\mathrm{b}}\left(y_{1}^{+}, t\right)-C_{\mathrm{b}}\left(y_{1}^{-}, t\right)\right)
\end{aligned}
$$




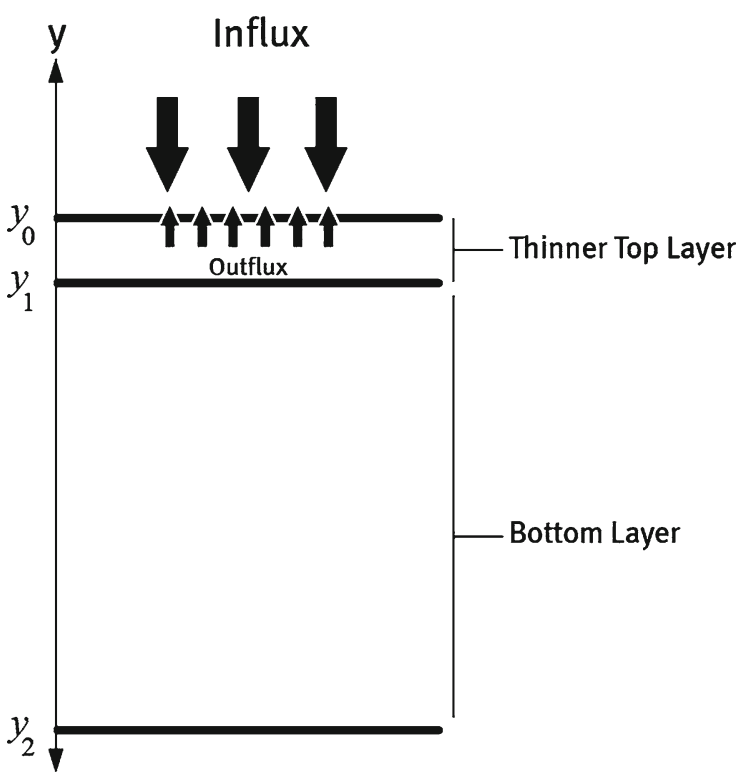

Fig. 3 One-dimensional configuration of the printing belt

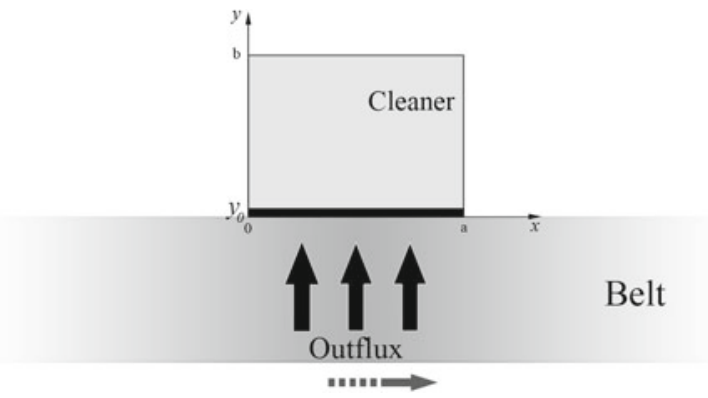

Fig. 4 Two-dimensional configuration of the cleaner

$$
\kappa_{2}\left[\frac{\partial C_{\mathrm{b}}}{\partial y}\right]_{y=y_{2}}=0,
$$

In the mathematical description, $\Theta(t)$ represents both the wax influx in the fuse nip at time intervals $(n-1) T_{\mathrm{p}}<t<$ $(n-1) T_{\mathrm{p}}+T_{\text {if }}$ and the wax outflux in the cleaner at time intervals $(n-1) T_{\mathrm{p}}+T_{\text {of }}<t<(n-1) T_{\mathrm{p}}+T_{\text {of }}+\Delta T_{\text {of }}$. The parameter $h_{12}$ is the inverse contact resistance; we refer to the works of Bourouga and Bardon [6] and Yeh and Wen [7] regarding this parameter. The higher $h_{12}$ the better the contact between layer one and layer two; the + and - symbols denote the limits

$$
f\left(y^{ \pm}\right)=\lim _{\delta \rightarrow 0} f(y \pm \delta)
$$

Since the cleaner does not move, we describe it by a fixed Cartesian reference system, with $\mathbf{e}_{x}$ being the direction of contact and $\mathbf{e}_{y}$ the direction normal to the belt; see Fig. 4. We assume that the wax concentration $C_{\mathrm{c}}$ is independent of $z$, so that the evolution of $C_{\mathrm{c}}$ is described by the model

$\frac{\partial C_{\mathrm{c}}}{\partial t}=\kappa_{\mathrm{c}}\left(\frac{\partial^{2} C_{\mathrm{c}}}{\partial x^{2}}+\frac{\partial^{2} C_{\mathrm{c}}}{\partial y^{2}}\right)-\alpha C_{\mathrm{c}}, \quad 0<x<a, \quad 0<y<b, \quad t>0$,

where the boundary conditions for the wax concentration $C_{\mathrm{c}}$ of the cleaner are given by

$\kappa_{\mathrm{c}}\left[\frac{\partial C_{\mathrm{c}}}{\partial x}\right]_{x=0}=\kappa_{\mathrm{c}}\left[\frac{\partial C_{\mathrm{c}}}{\partial x}\right]_{x=a}=0$

$\kappa_{\mathrm{c}}\left[\frac{\partial C_{\mathrm{c}}}{\partial y}\right]_{y=0}=\Psi_{\mathrm{c}}(t)$

$\kappa_{\mathrm{c}}\left[\frac{\partial C_{\mathrm{c}}}{\partial y}\right]_{y=b}=0$

Here, the dimensions of the cleaner are given by $a$ and $b$, and $\kappa_{\mathrm{c}}$ and $\alpha$ represent the cleaner diffusiveness and the wax decomposition rate, respectively. The wax influx is represented by $\Psi_{\mathrm{c}}(t)$.

In (3), we introduce the wax flux $\Theta(t)$. It is decomposed as

$$
\Theta(t)=-\Phi(t)+\Psi_{\mathrm{b}}(t), \quad t>0
$$


where $\Phi(t)$ describes the wax influx, a step function,

$\Phi(t)=\left\{\begin{array}{l}\left.\Phi, \quad(n-1) T_{\mathrm{p}}\right)<t<(n-1) T_{\mathrm{p}}+T_{\mathrm{if}}, \quad n=1,2, \ldots \\ 0, \text { else }\end{array}\right.$

The function $\Psi_{\mathrm{b}}(t)$ is modeled as

$\Psi_{\mathrm{b}}(t)=\left\{\begin{array}{l}h_{\mathrm{R}}\left(\bar{C}_{\mathrm{b}}\left((n-1) T_{\mathrm{p}}\right)-\bar{C}_{\mathrm{c}}\left((n-1) T_{\mathrm{p}}\right)\right), \\ \quad(n-1) T_{\mathrm{p}}+T_{\mathrm{of}}<t<(n-1) T_{\mathrm{p}}+T_{\mathrm{of}}+\Delta T_{\mathrm{of}}, \\ n=1,2, \ldots \\ 0, \quad \text { else. }\end{array}\right.$

Here, $\bar{C}_{\mathrm{b}}\left((n-1) T_{\mathrm{p}}\right)$ and $\bar{C}_{\mathrm{c}}\left((n-1) T_{\mathrm{p}}\right)$ denote the averaged wax concentration of the belt and of the cleaner, respectively, at the $n$th revolution cycle.

We write $\Psi_{n}=h_{\mathrm{R}}\left(\bar{C}_{\mathrm{b}}\left((n-1) T_{\mathrm{p}}\right)-\bar{C}_{\mathrm{c}}\left((n-1) T_{\mathrm{p}}\right)\right)$. We note that after every revolution of the belt, we should update the wax outflux $\Psi_{n}$. The wax influx of the cleaner is given by the step function

$\Psi_{\mathrm{c}}(t)=\Psi_{n}, \quad(n-1) T_{\mathrm{p}}<t \leq n T_{\mathrm{p}}, \quad n=1,2, \ldots$

\section{Solution procedure}

Since it is necessary to calculate the wax concentration for a great number of belt revolutions, we do not discretize time. With respect to the spatial variable $y$, we describe the functional relation of the solution in terms of well chosen cubic splines, where node point refer to space discretization. We make full use of the "cyclic" nature of the problem. We consider two basic problems:

1. The belt

$$
\begin{aligned}
& \frac{\partial A_{\mathrm{b}}}{\partial t}=\kappa_{i} \frac{\partial^{2} A_{\mathrm{b}}}{\partial y^{2}}, \quad y_{i-1}<y<y_{i}, \quad t>0, \quad i=1,2 \\
& \kappa_{1}\left[\frac{\partial A_{\mathrm{b}}}{\partial y}\right]_{y=y_{0}}=-1, \quad t>0 \\
& \kappa_{2}\left[\frac{\partial A_{\mathrm{b}}}{\partial y}\right]_{y=y_{2}}=0, \quad t>0 \\
& \kappa_{1}\left[\frac{\partial A_{\mathrm{b}}}{\partial y}\right]_{y=y_{1}^{-}}=\kappa_{2}\left[\frac{\partial A_{\mathrm{b}}}{\partial y}\right]_{y=y_{1}^{+}}, \quad t>0 \\
& \kappa_{1}\left[\frac{\partial A_{\mathrm{b}}}{\partial y}\right]_{y=y_{1}^{-}}=h_{12}\left(A_{\mathrm{b}}\left(y_{1}^{+}, t\right)-A_{\mathrm{b}}\left(y_{1}^{-}, t\right)\right) \\
& A_{\mathrm{b}}(y, t)=0, \quad t \leq 0, \quad y_{0}<y<y_{2}
\end{aligned}
$$

2. The cleaner

$$
\begin{aligned}
& \frac{\partial A_{\mathrm{c}}}{\partial t}=\kappa_{\mathrm{c}}\left(\frac{\partial^{2} A_{\mathrm{c}}}{\partial x^{2}}+\frac{\partial^{2} A_{\mathrm{c}}}{\partial y^{2}}\right)-\alpha A_{\mathrm{c}}, \quad 0<x<a, \quad y_{0}-b<y<y_{0}, \quad t>0 \\
& \kappa_{\mathrm{c}}\left[\frac{\partial A_{\mathrm{c}}}{\partial x}\right]_{x=0}=\kappa_{\mathrm{c}}\left[\frac{\partial A_{\mathrm{c}}}{\partial x}\right]_{x=a}=0, \quad t>0 \\
& \kappa_{\mathrm{c}}\left[\frac{\partial A_{\mathrm{c}}}{\partial y}\right]_{y=y_{0}}=1, \quad t>0 \\
& \kappa_{\mathrm{c}}\left[\frac{\partial A_{\mathrm{c}}}{\partial y}\right]_{y=y_{0}-b}=0, \quad t>0 \\
& A_{\mathrm{c}}(x, y, t)=0, \quad t \leq 0
\end{aligned}
$$


In term of $A_{\mathrm{b}}$ and $A_{\mathrm{c}}$ we can represent $C_{\mathrm{c}}$ and $C_{\mathrm{b}}$ as

$$
\begin{aligned}
C_{\mathrm{b}}(y, t)= & \Phi \sum_{n=1}^{\infty}\left[A_{n}\left(y, t-(n-1) T_{\mathrm{p}}\right)-A_{\mathrm{b}}\left(y, t-(n-1) T_{\mathrm{p}}-T_{\mathrm{of}}\right)\right] \\
& -\sum_{n=1}^{\infty} \Psi_{n}\left[A_{\mathrm{b}}\left(y, t-(n-1) T_{\mathrm{p}}-T_{\mathrm{of}}\right)-A_{\mathrm{b}}\left(y, t-(n-1) T_{\mathrm{p}}-T_{\mathrm{of}}-\Delta T_{\mathrm{of}}\right)\right] \\
C_{\mathrm{c}}(y, t)= & \sum_{n=1}^{\infty} \Psi_{n}\left[A_{\mathrm{c}}\left(x, y, t-(n-1) T_{\mathrm{p}}\right)-A_{c}\left(x, y, t-n T_{\mathrm{p}}\right)\right] \\
= & \sum_{n=1}^{\infty}\left(\Psi_{n}-\Psi_{n-1}\right) A_{\mathrm{c}}\left(x, y, t-(n-1) T_{\mathrm{p}}\right)
\end{aligned}
$$

Clearly $A_{\mathrm{c}}$ is independent of the variable $x$ and can be written as

$$
\begin{aligned}
& A_{\mathrm{c}, \mathrm{st}}(y)=\sqrt{\frac{\kappa_{\mathrm{c}}}{\alpha}} \frac{1}{\sinh \left(b \sqrt{\frac{\alpha}{\kappa_{\mathrm{c}}}}\right)} \cosh \left(\sqrt{\frac{\alpha}{\kappa_{\mathrm{c}}}}\left(y-y_{0}+b\right)\right) \\
& A_{\mathrm{c}}(x, y, t)=A_{\mathrm{c}, \mathrm{st}}(y)+A_{\mathrm{c}, \mathrm{tr}}(y, t)
\end{aligned}
$$

where with a straightforward separation of variables technique

$$
A_{\mathrm{c}, \operatorname{tr}}(y, t)=\sum_{l=0}^{\infty} \gamma_{l} \exp \left(-\left(\left(\frac{\pi l}{b}\right)^{2}+\alpha\right) t\right) \cos \left(\pi l\left(\frac{y-y_{0}+b}{b}\right)\right)
$$

Thus,

$$
\begin{aligned}
& \gamma_{0}=\frac{1}{b} \int_{0}^{b}\left[A_{\mathrm{c}}(y, 0)-A_{\mathrm{c}, \mathrm{st}}(y)\right] \mathrm{d} y \\
& \gamma_{l}=\frac{2}{b} \int_{0}^{b}\left[A_{\mathrm{c}}(y, 0)-A_{\mathrm{c}, \mathrm{st}}(y)\right] \cos \left(\pi l\left(\frac{y-y_{0}+b}{b}\right)\right) \mathrm{d} y
\end{aligned}
$$

The basic solution $A_{\mathrm{b}}(y, t)$ is split into three parts

$A_{\mathrm{b}}(y, t)=A_{\mathrm{b}, 1}(y, t)+A_{\mathrm{b}, 2}(y, t)+A_{\mathrm{b}, 3}(y, t)$,

where $A_{\mathrm{b}, 1}(y, t)$ and $A_{\mathrm{b}, 2}(y, t)$ are determined analytically and chosen such that they create homogenous boundary conditions. Then, $A_{\mathrm{b}, 3}$ satisfies a inhomogeneous diffusion equation with homogeneous boundary conditions. We discretize the elliptic operator that describes the multi-layer diffusion problem, by choosing appropriate basis functions in its domain. For that we introduce an artificial layer $\left(y_{0}, y_{01}\right)$ with $y_{01} \leq y_{1} / 2$ such that the diffusion problem

$$
\begin{aligned}
& \frac{\partial A}{\partial t}=\kappa_{1} \frac{\partial^{2} A}{\partial y^{2}}, \quad y_{0}<y<y_{01}, \quad t>0 \\
& A(y, 0)=0 \\
& \kappa_{1}\left[\frac{\partial A}{\partial y}\right]_{y=y_{01}}=0 \\
& \kappa_{1}\left[\frac{\partial A}{\partial y}\right]_{y=y_{0}}=-1
\end{aligned}
$$

is well approximated by the solution 
$A_{\mathrm{b}, 1}(y, t)=\frac{t}{y_{01}-y_{0}}+\frac{1}{\kappa_{1}}\left(y_{01}-y_{0}\right) F(y, t), \quad t \geq 0$.

where $F(y, t)$ is a function with order of magnitude one and average zero. Due to the fact that the physical layer is very thin such that $\left(y_{01}-y_{0}\right) \ll 1$, the solution can be written as the average solution as follows:

$A_{\mathrm{b}, 1}(y, t)= \begin{cases}\frac{t}{y_{01}-y_{0}}, & t \geq 0 \\ 0, & t<0 .\end{cases}$

Then, we choose a next artificial layer $\left(y_{01}, y_{02}\right)$ with $y_{02}-y_{01} \approx y_{01}-y_{1}$, so that the function $A_{\mathrm{b}, 2}(y, t)$ satisfies

$\kappa_{1}\left[\frac{\partial A_{\mathrm{b}, 2}}{\partial y}\right]_{y=y_{01}^{-}}=\kappa_{1}\left[\frac{\partial A_{\mathrm{b}, 2}}{\partial y}\right]_{y=y_{01}^{+}}$

$A_{\mathrm{b}, 2}\left(y_{01}^{+}, t\right)-A_{\mathrm{b}, 2}\left(y_{01}^{-}, t\right)=A_{\mathrm{b}, 1}\left(y_{01}^{-}, t\right)$

We take

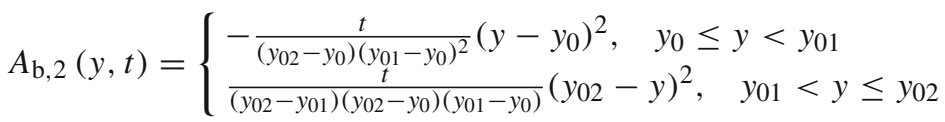

By the choice of $A_{\mathrm{b}, 1}$ and $A_{\mathrm{b}, 2}$, the third component $A_{\mathrm{b}, 3}$ satisfies an inhomogeneous diffusion equation and homogenous boundary and interface conditions

$$
\begin{aligned}
& \frac{\partial A_{\mathrm{b}, 3}}{\partial t}=\kappa_{1} \frac{\partial^{2} A_{\mathrm{b}, 3}}{\partial y^{2}}+Q(y, t), \quad y_{0}<y<y_{1}, \quad t>0 \\
& \frac{\partial A_{\mathrm{b}, 3}}{\partial t}=\kappa_{2} \frac{\partial^{2} A_{\mathrm{b}, 3}}{\partial y^{2}}, \quad y_{1}<y<y_{2}, \quad t>0 \\
& \kappa_{1}\left[\frac{\partial A_{\mathrm{b}, 3}}{\partial y}\right]_{y=y_{0}}=0, \quad \kappa_{2}\left[\frac{\partial A_{\mathrm{b}, 3}}{\partial y}\right]_{y=y_{2}}=0, \quad t>0 \\
& \kappa_{1}\left[\frac{\partial A_{\mathrm{b}, 3}}{\partial y}\right]_{y=y_{1}^{-}}=\kappa_{2}\left[\frac{\partial A_{\mathrm{b}, 3}}{\partial y}\right]_{y=y_{1}^{+}}, \quad t>0 \\
& \kappa_{1}\left[\frac{\partial A_{\mathrm{b}, 3}}{\partial y}\right]_{y=y_{1}^{-}}=h_{12}\left(A_{\mathrm{b}, 3}\left(y_{1}^{+}, t\right)-A_{\mathrm{b}, 3}\left(y_{1}^{-}, t\right)\right), \quad t>0 \\
& A_{\mathrm{b}, 3}(y, t)=0, \quad y_{0}<y<y_{1}, \quad t \leq 0
\end{aligned}
$$

Here,

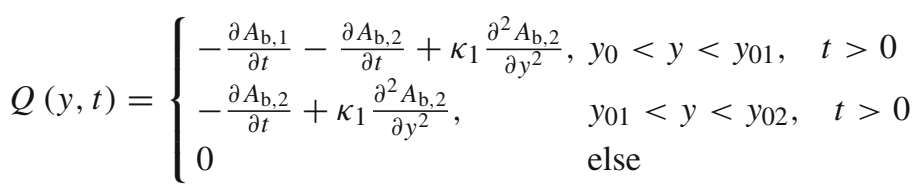

The solution $A_{\mathrm{b}, 3}$ can be written in the form of an expansion

$$
A_{\mathrm{b}, 3}(y, t)=\sum_{j=1}^{\infty} c_{j}(t) v_{j}(y)
$$

Here, $v_{j}(y)$ are the eigenfunctions of an associated coercive operator, a second-order differential operator with associated interface and boundary conditions. With the corresponding eigenvalues denoted by $\lambda_{j}$, we have

$c_{j}(t)=\int_{0}^{t} \mathrm{e}^{-\lambda_{j}(t-\tau)} q_{j}(\tau) \mathrm{d} \tau$

and 
$q_{j}(t)=\int_{y_{0}}^{y_{02}} Q(y, t) v_{j}(y) \mathrm{d} y$

under the normalization

$$
\int_{y_{0}}^{y_{2}} v_{j}(y)^{2} \mathrm{~d} y=1
$$

\section{Numerical implementation}

For the third component $A_{\mathrm{b}, 3}$, we develop a numerical approach which is partly analytic $[8,9]$. We only discretize with respect to the position variable $y$ and leave time $t$ a continuous variable. In the description of our approach, we take slightly more general point of departure. For that we define a positive Hermitian operator in a suitable Hilbert space with an essentially compact inverse. The operator is related to a generalized Sturm-Liouville problem and so has a complete set of orthogonal eigenfunctions with positive eigenvalue. The numerical approach is dedicated to the computation of these eigenfunctions and corresponding eigenvalues.

We consider an interval $\left(\eta_{0}, \eta_{M}\right)$ that is disjointly divided in $M$ subintervals

$\left(\eta_{0}, \eta_{M}\right) \stackrel{\circ}{=} \bigcup_{i=1}^{M}\left(\eta_{i-1}, \eta_{i}\right)$

On the Hilbert space $\mathcal{L}_{2}\left(y_{0}, y_{M}\right)$ the second-order differential operator $\mathfrak{F}$ is defined by

$[\mathfrak{F} u]_{\left(y_{i}, y_{i+1}\right)}=-\kappa_{i}\left[\frac{\partial^{2} u}{\partial y^{2}}\right]_{\left(y_{i}, y_{i+1}\right)}, \quad i=1, \ldots, M$,

where the operator $\mathfrak{F}$ has definition domain $\operatorname{dom}(\mathfrak{F})$, the dense subspace of all $u \in \mathcal{L}_{2}\left(y_{0}, y_{M}\right)$ that have the property as the following:

$[u]_{\left(\eta_{i-1}, \eta_{i}\right)} \in \mathcal{H}_{2,2}\left(\eta_{i-1}, \eta_{i}\right)$

and that satisfy the boundary and interface conditions

$\kappa_{i}\left[\frac{\partial u}{\partial y}\right]_{y=\eta_{i}^{-}}=\kappa_{i+1}\left[\frac{\partial u}{\partial y}\right]_{y=\eta_{i}^{+}}$

$\kappa_{i}\left[\frac{\partial u}{\partial y}\right]_{y=\eta_{i}^{-}}=h_{i}\left(u\left(y_{i}^{+}\right)-u\left(y_{i}^{-}\right)\right)$,

$\kappa_{0}\left[\frac{\partial u}{\partial y}\right]_{y=\eta_{0}}=0, \quad \kappa_{M}\left[\frac{\partial u}{\partial y}\right]_{y=\eta_{M}}=0$

We note that $h_{i}=\infty$ means $u\left(y_{i}^{+}\right)=u\left(y_{i}^{-}\right)$. By writing

$\langle u, v\rangle=\sum_{i=1}^{M} \int_{\eta_{i-1}}^{\eta_{i}} u v^{*} \mathrm{~d} y$

we see that $\mathcal{F}$ is a positive Hermitian operator, i.e.,

$\langle\mathcal{F} u, v\rangle=\langle u, \mathcal{F} v\rangle, \quad\langle u, \mathcal{F} v\rangle \geq 0$

For each $\alpha>0, \alpha I+\mathcal{F}$ is a strictly positive operator with a compact inverse. Thus, $\mathfrak{F}$ represents a generalized Sturm-Liouville operator. There is a complete orthonormal set of eigenfunctions $\left(v_{j}\right)_{j=1}^{\infty}$ contained in $\operatorname{dom}(\mathfrak{F})$ and corresponding eigenvalues $\left(\lambda_{j}\right)$ with $\lambda_{j} \geq 0$ and

$\lim _{j \rightarrow \infty} \frac{1}{\lambda_{j}}=0$ 
such that

$\mathfrak{F} u=\sum_{j=1}^{\infty} \lambda_{j}\left(u, v_{j}\right)_{\mathcal{L}_{2}} v_{j}$.

The evolution equation

$\frac{\mathrm{d} u}{\mathrm{~d} t}+\mathfrak{F} u=Q(t)$

where

$u:(0, \infty) \rightarrow \operatorname{dom}(\mathcal{F})$

and

$Q:(0, \infty) \rightarrow \mathcal{L}\left(\eta_{0}, \eta_{M}\right)$

satisfies

$u(t)=\sum_{j=1}^{\infty} c_{j}(t) v_{j}$

with

$c_{j}(t)=\left(\gamma_{j}+\int_{0}^{t} q_{n, j}(\tau) \mathrm{e}^{\lambda_{j} \tau} \mathrm{d} \tau\right) \mathrm{e}^{-\lambda_{j} t}$

$q_{j}(t)=\left(Q(t), v_{j}\right)_{\mathcal{L}_{2}}$

Our effort is put into the calculation of the eigenfunctions and eigenvalues. For that we construct a set of basic functions $\mathfrak{B}_{k}, k=1, \ldots, K$ in $\operatorname{dom}(\mathfrak{F})$ and approximate the first $K$ eigenfunctions by

$v_{j}=\sum_{k=1}^{K} \beta_{j k} \mathfrak{B}_{k}, \quad j=1, \ldots, K$.

The eigenvalue equation

$\mathfrak{F} v_{j}=\lambda_{j} v_{j}$

is replaced by the generalized matrix eigenvalue equation

$\mathbf{F} \beta_{j}=\lambda_{j} \mathbf{G} \beta_{j}$

where

$$
\begin{aligned}
& \mathbf{F}=\left(\left(\mathfrak{F} \mathfrak{B}_{k}, \mathfrak{B}_{l}\right)_{\mathcal{L}_{2}}\right)_{k, l=1}^{K} \\
& \mathbf{G}=\left(\left(\mathfrak{B}_{k}, \mathfrak{B}_{l}\right) \mathcal{L}_{2}\right)_{k, l=1}^{K} \\
& \beta_{j}=\left(\beta_{j k}\right)_{k=1}^{K}, \quad j=1, \ldots, K
\end{aligned}
$$

In order to construct the basis function $\mathcal{B}_{k}$ we use four elementary third degree polynomials

$\varphi_{1}(\hat{y})=(2 \hat{y}+1)(\hat{y}-1)^{2}$

$\varphi_{2}(\hat{y})=\hat{y}(\hat{y}-1)^{2}$

$\varphi_{3}(\hat{y})=(3-2 \hat{y}) \hat{y}^{2}$

$\varphi_{4}(\hat{y})=-(1-\hat{y}) \hat{y}^{2}$

We note that

$\varphi_{1}(1)=\varphi_{1}^{\prime}(1)=\varphi_{2}(1)=\varphi_{2}^{\prime}(1)=0$ 
and

$\varphi_{1}(0)=1, \quad \varphi_{1}^{\prime}(0)=0, \quad \varphi_{2}(0)=0, \quad \varphi_{2}^{\prime}(0)=1$.

The basis functions are now defined as

$\mathfrak{B}_{1}(y)= \begin{cases}\varphi_{1}\left(\frac{y-\eta_{0}}{\eta_{1}-\eta_{0}}\right), & \eta_{0} \leq y<\eta_{1} \\ 0, & \eta_{1} \leq y \leq \eta_{M}\end{cases}$

$\mathfrak{B}_{2 M}(y)= \begin{cases}\varphi_{3}\left(\frac{y-\eta_{M-1}}{\eta_{M}-\eta_{M-1}}\right), & \eta_{M-1}<y \leq \eta_{M} \\ 0, & \eta_{0} \leq y \leq \eta_{M-1} .\end{cases}$

For node point $\eta_{i}$, we introduce two basis functions $\mathcal{B}_{2 i+1}$ and $\mathcal{B}_{2 i}$ of the form

$\mathfrak{B}_{l}(y)= \begin{cases}\delta_{3}^{i-1} \varphi_{3}\left(\frac{y-\eta_{i-1}}{\eta_{i}-\eta_{i-1}}\right)+\delta_{4}^{i-1} \varphi_{4}\left(\frac{y-\eta_{i-1}}{\eta_{i}-\eta_{i-1}}\right), & \eta_{i-1} \leq y<\eta_{i} \\ \delta_{1}^{i} \varphi_{1}\left(\frac{y-\eta_{i-1}}{\eta_{i}-\eta_{i-1}}\right)+\delta_{2}^{i} \varphi_{2}\left(\frac{y-\eta_{i-1}}{\eta_{i}-\eta_{i-1}}\right), & \eta_{i} \leq y<\eta_{i+1} \\ 0, & \text { else }\end{cases}$

The function $\mathfrak{B}_{l}(y)$ satisfies the interface conditions at $y=\eta_{i-1}$ and at $y=\eta_{i+1}$. In order to satisfy the interface conditions at $y=\eta_{i}$ the coefficients $\delta_{1}^{i}, \delta_{2}^{i}, \delta_{3}^{i-1}$, and $\delta_{4}^{i-1}$ should satisfy

$\delta_{4}^{i-1}=-\frac{\kappa_{i+1}}{\kappa_{i}} \frac{\eta_{i}-\eta_{i-1}}{\eta_{i+1}-\eta_{i}} \delta_{2}^{i}$

$\delta_{4}^{i-1}=\frac{h_{i}\left(\eta_{i}-\eta_{i-1}\right)}{\kappa_{i}}\left(\delta_{1}^{i}-\delta_{3}^{i-1}\right)$

The two equations have two independent solutions; we take

$\delta_{3}^{i-1}=\xi_{i}, \quad \delta_{4}^{i-1}=1$

$\delta_{1}^{i}=\xi_{i+1}, \quad \delta_{2}^{i}=-\frac{\kappa_{i}}{\kappa_{i+1}} \frac{\eta_{i+1}-\eta_{i}}{\eta_{i}-\eta_{i-1}}$

where

$\xi_{i+1}=\xi_{i}+\frac{\kappa_{i}}{h_{i}\left(\eta_{i}-\eta_{i-1}\right)}, \quad \xi_{1}=1$

and

$\delta_{3}^{i-1}=1, \quad \delta_{4}^{i-1}=0$

$\delta_{1}^{i}=1, \quad \delta_{2}^{i}=0$

In order to increase the spatial resolution and therewith the accuracy of the numerical approximation, we can introduce artificial layers and artificial interface condition with inverse contact resistance infinity. The number of layers determines the number of eigenvalues and eigenfunctions numerically calculated. As a rule of thumb, given $2 M$ basis functions, the first $M$ eigenvalues, $\lambda_{1}<\cdots<\lambda_{M}$, are well-determined. Of course, the more we want to zoom into the transient behavior of the system, the more eigenvalues, in increasing order, need to be calculated.

The belt system that we analyzed for this article, has two physical layers $\left(y_{0}, y_{1}\right)$ and $\left(y_{1}, y_{2}\right)$. The first layer is divided into $M_{1}$ parts with $M_{1} \geq 2$. The second layer is divided into $M_{2}$ parts. At best all sublayers should have the same time scale related, i.e.,

$$
\left(\frac{y_{1}-y_{0}}{M_{1}}\right)^{2} \frac{1}{\kappa_{1}} \simeq\left(\frac{y_{2}-y_{1}}{M_{2}}\right)^{2} \frac{1}{\kappa_{2}}
$$

In our numerical implementation, we took $M_{1}=2$ and $M_{2}=5$. 
Table 1 Lab model printer: numerical values for the properties of the belt
Table 2 Lab model printer: numerical values for the properties of the cleaner

\begin{tabular}{lll}
\hline Parameter & Units & Value \\
\hline Length & $\mathrm{m}$ & 0.437 \\
Thickness & $\mathrm{m}$ & $1.35 \times 10^{-3}$ \\
Length of the area for the wax influx & $\mathrm{m}$ & $7 \times 10^{-3}$ \\
Distance between the influx point and the cleaner & $\mathrm{m}$ & 0.128 \\
Velocity of the belt in high mode (high speed) & $\mathrm{m} \cdot \mathrm{s}^{-1}$ & 0.485 \\
Velocity of the belt in low mode $($ low speed $)$ & $\mathrm{m} \cdot \mathrm{s}^{-1}$ & 0.05 \\
Diffusivity constant for layer 1 $\left(\kappa_{1}\right)$ & $\mathrm{m}^{2} \mathrm{~s}^{-1}$ & $10^{-10}$ \\
Diffusivity constant for layer 2 $\left(\kappa_{1}\right)$ & $\mathrm{m}^{2} \mathrm{~s}^{-1}$ & $4 \times 10^{-10}$ \\
Wax influx $(\Phi)$ & $\mathrm{kg} \cdot \mathrm{m}^{-2} \mathrm{~s}^{-1}$ & $1.7 \times 10^{-5}$ \\
Inverse contact resistance $\left(h_{\mathrm{i}}\right)$ & $\mathrm{m} \cdot \mathrm{s}^{-1}$ & $10^{5}$ \\
\hline
\end{tabular}

Table 3 Lab model printer: numerical values for the time parameters

\begin{tabular}{lll}
\hline Parameter & Units & Value \\
\hline Length & $\mathrm{m}$ & $4.5 \times 10^{-3}$ \\
Thickness & $\mathrm{m}$ & $2 \times 10^{-3}$ \\
Diffusivity constant $\left(\kappa_{\mathrm{c}}\right)$ & $\mathrm{m}^{2} \mathrm{~s}^{-1}$ & $8 \times 10^{-9}$ \\
Decomposition rate at $100^{\circ} \mathrm{C}(\alpha)$ & $\mathrm{s}^{-1}$ & $2.422 \times 10^{-5}$ \\
Inverse contact resistance $\left(h_{\mathrm{R}}\right)$ & $\mathrm{m} \cdot \mathrm{s}^{-1}$ & $10^{-7}$ \\
\hline
\end{tabular}

\begin{tabular}{ll}
\hline Time parameter & Value (s) \\
\hline$T_{\text {if }}$ & 0.0144 \\
$T_{\text {of }}$ & 0.2639 \\
$\triangle T_{\text {of }}$ & 0.0093 \\
$T_{\mathrm{p}}$ & 0.894 \\
\hline
\end{tabular}

\section{Numerical results}

The capacity of the printer in a high-speed mode for printing A4 size paper is 6,000 copies per hour, or approximately 1.6 copies per round.

In the generation of numerical results, we consider a two layer belt with a top layer of $0.15 \mathrm{~mm}$ and a bottom layer of $1.20 \mathrm{~mm}$. We divide the top layer into two sublayers one of $0.07 \mathrm{~mm}$ and the other one of $0.08 \mathrm{~mm}$. The bottom layer is divided into five sublayers, one with a thickness of $0.20 \mathrm{~mm}$ and the other ones with a thickness of $0.25 \mathrm{~mm}$. The contact between the belt sublayers is almost perfect, $h_{i}=10^{5} \mathrm{~m} / \mathrm{s}$, while the contact between cleaner and belt is not ideal, $h_{R}=10^{-7} \mathrm{~m} / \mathrm{s}$. In Tables 1 and 2 , the values of all process and material parameters as delivered by Oce are presented. The time parameters values are shown in Table 3 . In this section, first we validate our model by comparison with data obtained from measurement and present results that simulate two procedures of cleaning. Second, we study the sensitivity on the decomposition rate $\alpha$ and the inverse contact resistance $h_{\mathrm{R}}$ of the wax build up in the belt. 


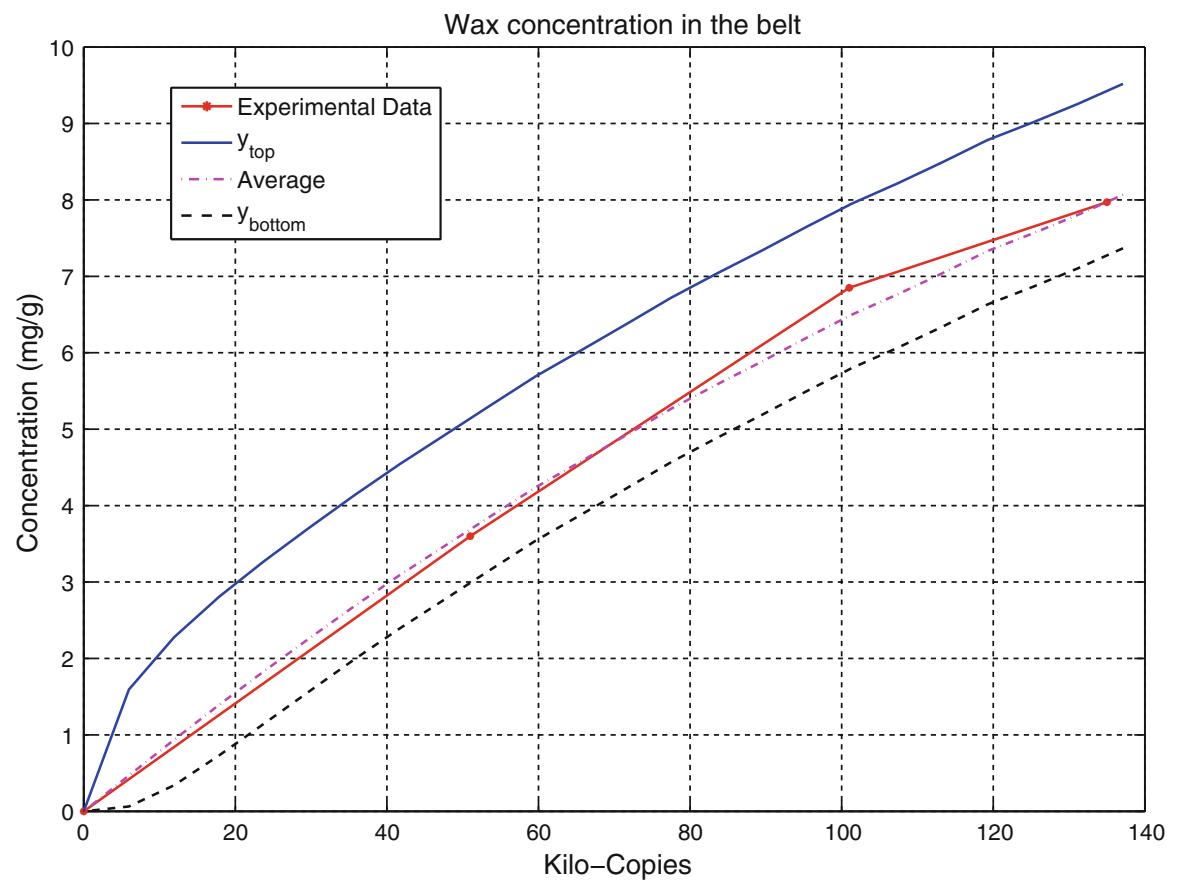

Fig. 5 The simulation result for 140,000 copies and comparison with the measurement data. The solid line and dashed line show the wax concentrations at the top and bottom, respectively. The average concentration, the dash-dotted line, matches the measurement data

\subsection{Simulation}

In Fig. 5, we compare the results obtained from measurement and the results obtained from the model. The plot shows that the numerically computed average wax concentration in the belt matches the one obtained from experiments very accurately. The difference between concentration at the top and bottom tends to become constant and equals $2 \%$. In the simulation, we took 140,000 copies of printing or 87,500 belt revolutions corresponding with a CPU-time of approximately $20 \mathrm{~min}$.

In Figs. 6 and 7, we consider 572,000 copies (357,500 rounds), where the cleaner is lifted up from the belt and cleaned after every 20,000 copies (12,500 rounds). During the lift up of the cleaner the printing process is stopped. The time evolution of the average wax concentration is not smooth. The wax concentration in the cleaner increases rapidly every round of 12,500 revolutions. We conclude that with the simulation an optimal cleaning strategy can be developed.

\subsection{Effect of inverse contact resistance and decomposition rate}

In this subsection, we investigate what is the most effective increase of decomposition rate or decrease of inverse contact resistance. We simulated $24 \mathrm{~h}$ of continuous process printing for each setting of the two parameters. We selected these parameters, because they can be adjusted in the real-world cleaning process.

In the first simulation, we have perfect contact and very bad contact between belt and cleaner $\left(h_{\mathrm{R}}=10^{-4} \mathrm{~m} / \mathrm{s}\right.$ and $h_{\mathrm{R}}=10^{-8} \mathrm{~m} / \mathrm{s}$, respectively) and vary the decomposition rate with $\alpha=10^{-7}, \ldots, 10^{-1} \mathrm{~s}^{-1}$; see Fig. 8. For the lowest decomposition rate, the cleaning process is still effective with a belt wax concentration of $80 \%$. However, for a very bad contact, the cleaning process is totally ineffective. 


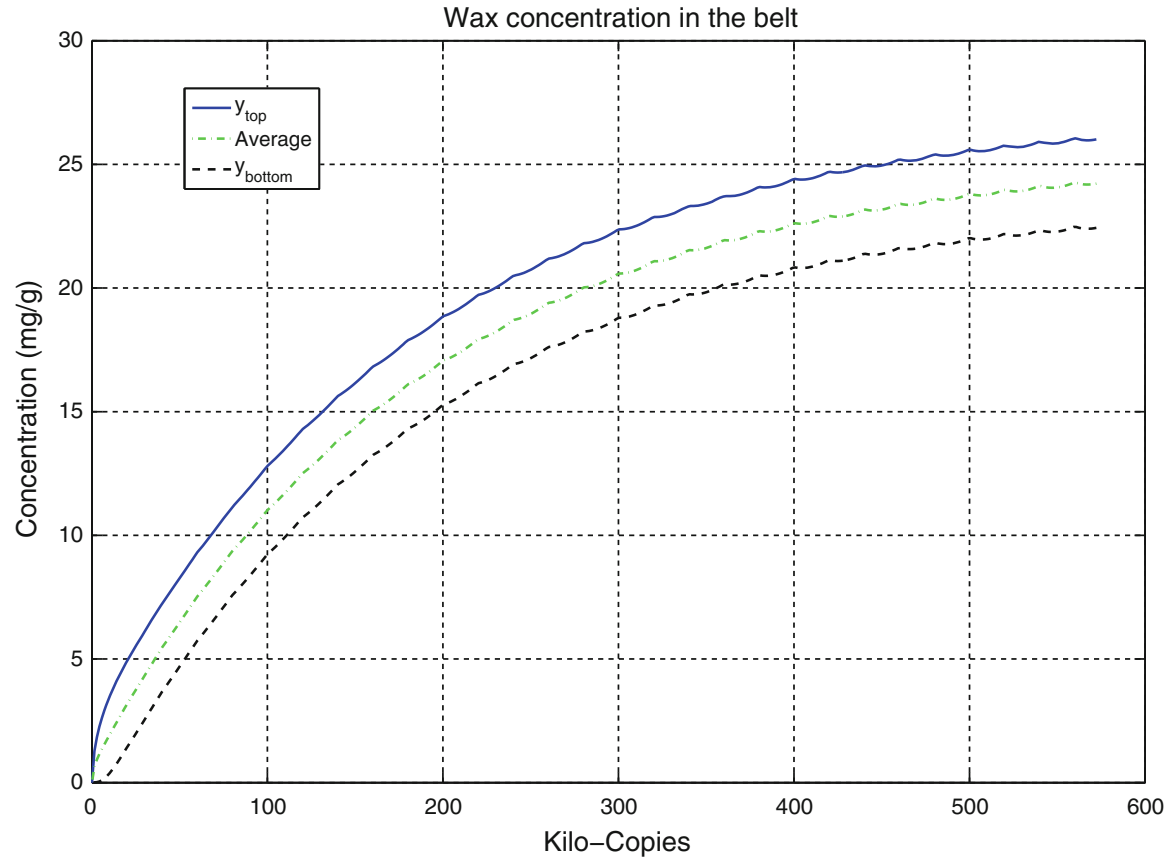

Fig. 6 The wax concentration profile in the belt for 572,000 copies prints. The solid line and dashed line show the wax concentrations at the top and bottom, respectively. The average concentration is depicted by the dashed-dotted line

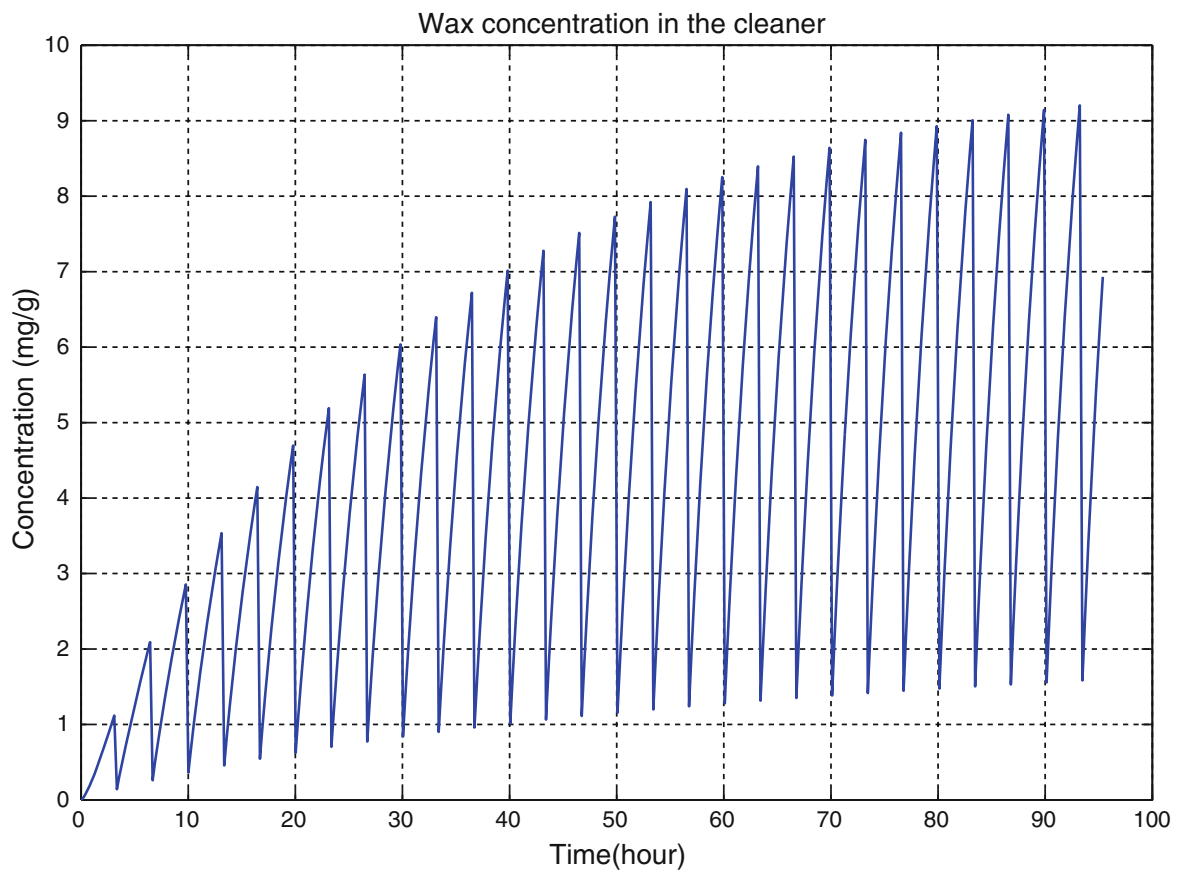

Fig. 7 The wax concentration profile in the cleaner for 572,000 copies prints or $95 \mathrm{~h}$ printing process 


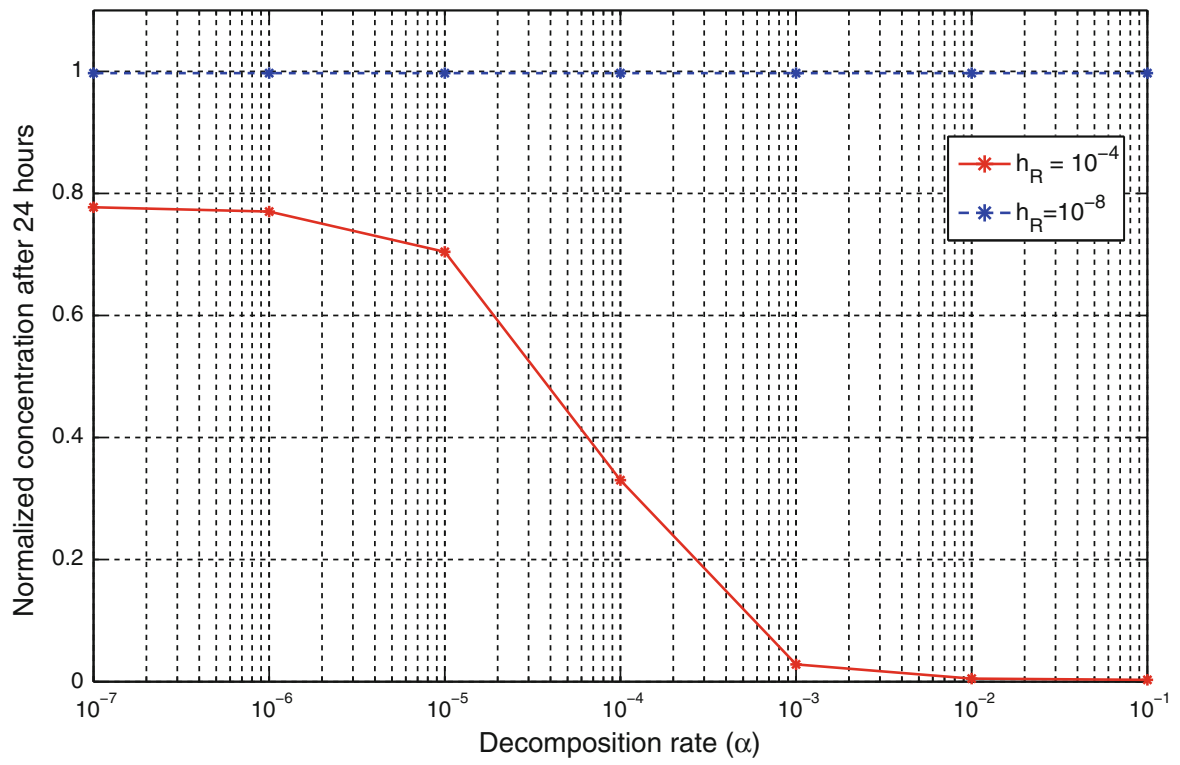

Fig. 8 Decomposition rate $(\alpha)$ versus normalized wax concentration in the belt after $24 \mathrm{~h}$. The dashed line depicts the case for inverse contact resistance $h_{\mathrm{R}}=10^{-8} \mathrm{~m} / \mathrm{s}$ and solid line for $h_{\mathrm{R}}=10^{-4} \mathrm{~m} / \mathrm{s}$

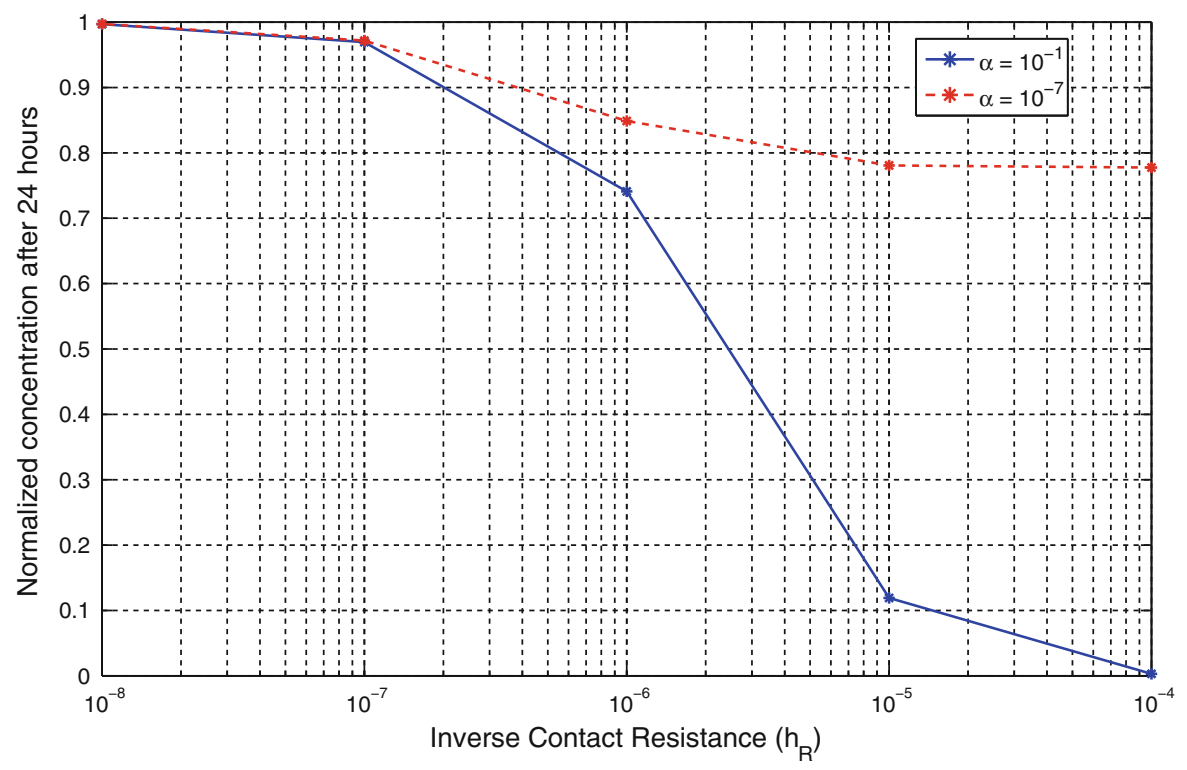

Fig. 9 Inverse contact resistance $\left(h_{\mathrm{R}}\right)$ versus normalized wax concentration in the belt after $24 \mathrm{~h}$. The dashed line depicts the case for decomposition rate $\alpha=10^{-7} \mathrm{~s}^{-1}$ and solid line for $\alpha=10^{-1} \mathrm{~s}^{-1}$

In the second simulation, we have a high-decomposition rate and a low-decomposition rate $\left(\alpha=10^{-1} \mathrm{~s}^{-1}\right.$ and $\alpha=10^{-8} \mathrm{~s}^{-1}$ respectively) and vary the inverse contact resistance $h_{\mathrm{R}}=10^{-8}, \ldots, 10^{-4} \mathrm{~m} / \mathrm{s}$; see Fig. 9 . We see that a high-inverse contact resistance $\left(h_{\mathrm{R}}<10^{-7} \mathrm{~s}^{-1}\right)$ leads to an ineffective cleaning process. 


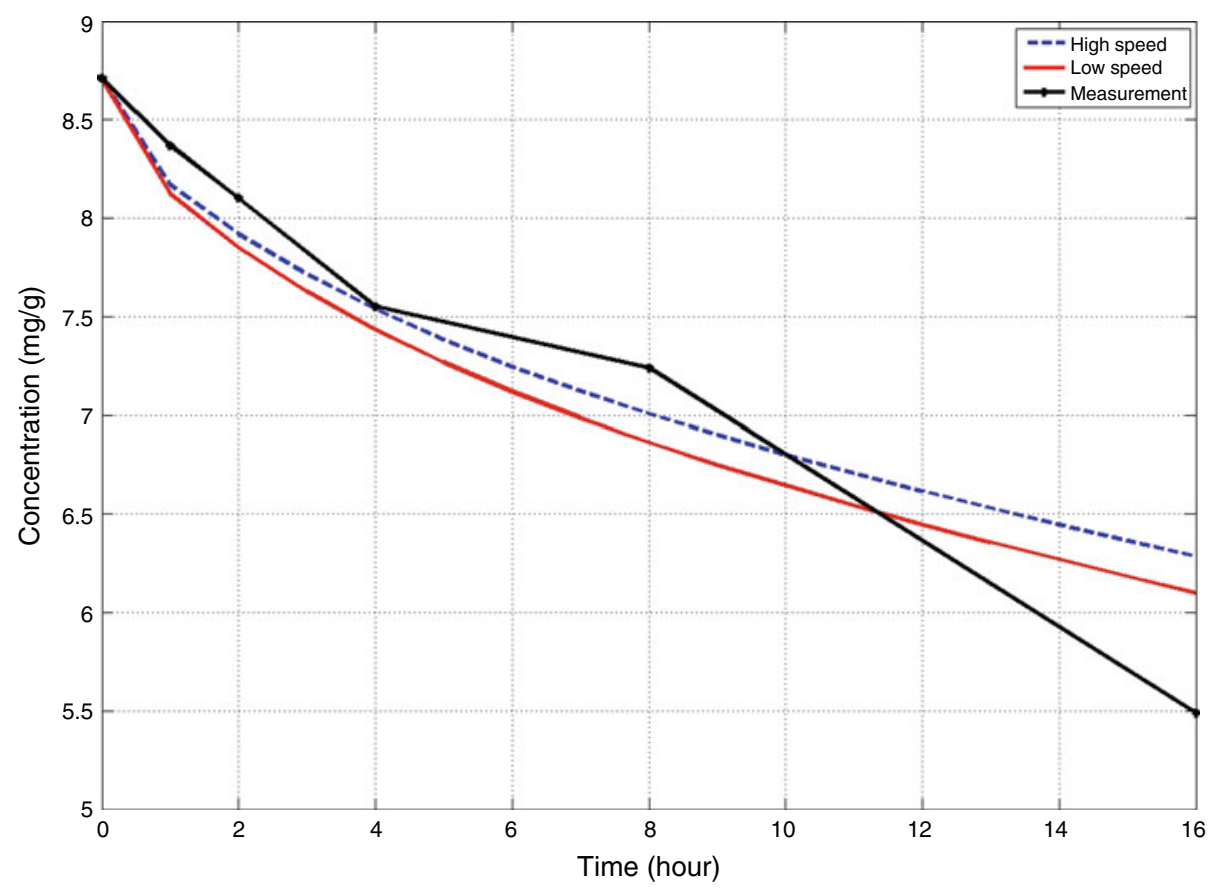

Fig. 10 The comparison of the cleaner effectiveness with respect to the speed configuration. The dashed line depicts the wax concentration in high-speed condition $(48.5 \mathrm{~cm} / \mathrm{s})$ and solid line is for the low- speed condition $(5 \mathrm{~cm} / \mathrm{s})$

\subsection{Effect of the belt speed}

We compare the wax build up for two belt speeds, a low speed of $5 \mathrm{~cm} / \mathrm{s}$ and a high speed of $48.5 \mathrm{~cm} / \mathrm{s}$; see Fig. 10. The results from simulation show that for the high-speed mode the wax concentration is higher than for the low-speed mode. However, the difference is not more than $2 \%$ and is therefore not significant.

\subsection{Computation speed}

Another aspect that is very important for any numerical simulation is the computation time. The present simulation tools at Océ are based on the finite element and finite volume method. With these tools, one round of process takes approximately $2 \mathrm{~h}$ to be processed. For comparison with our numerical tool, it takes approximately $20 \mathrm{~min}$ to simulate 90,000 rounds of wax diffusion process. This shows that the numerical method presented in this article is highly more effective and faster.

\section{Conclusions}

In this article, a numerical routine is described by which the wax build-up in a toner carrying belt can be simulated. The routine is based on a mathematical model that allows for a combination of an analytical and a numerical approach. The influx of wax is that slow a process that at least 20,000 copies of printing are required to see some effect. Thus, a standard approach based on time discretization and finite elements is inappropriate. Validation with respect to experimental data show perfect agreement. The conclusions from the model are that the contact resistance between belt and cleaner unit has more impact on the cleaning process than the decomposition rate. Also, the speed of the belt has negligible impact. Although the model is dedicated to wax diffusion in a belt with a cleaning unit 
at the top, similar models can be developed if the cleaning unit is positioned at the bottom of the belt, if the belt consists of more than two physical layers, or if more than one cleaning unit and more than one fuse nip are attached to the belt.

Of course the model can be made generic. Then, we describe a diffusion/convection process of a physical quantity in a closed loop transporting device, which moves with a constant speed and at which units are in contact that control influx or outflux. The assumption is that the speed of the transportation is that fast that diffusion along the direction of motion can be ignored and only diffusion in the transversal direction should be taken into account. In particular, the model can be used to describe the heat distribution in the belt due to heating (influx) at the fuse nip and cooling (outflux) at the image forming unit.

\section{Appendix}

In order to include wax saturation, the contact resistance can be modeled as a function of the concentration at the interface such that $h_{i}$ depends on $\left[C_{i}\right]_{y=y_{i}^{-}}$if $\left[C_{i}\right]_{y=y_{i}^{-}}>\left[C_{i+1}\right]_{y=y_{i}^{+}}$and $\left[C_{i+1}\right]_{y=y_{i}^{+}}$else. We propose a model to describe saturation in one of the layers. It comes down to letting the contact resistance depend on the concentrations at the interfaces. For sake of convenience, in the main part of the article, we let the parameters $h_{i}$ be constant.

The saturation problem appears if the inverse contact resistances on the interface $h_{i}$ leads to zero for a finite value of the wax concentration in the belt. When the wax concentration increases, the capability of the wax to penetrate the boundary layer decreases. In the case of a multi-layered belt, this condition influences the inverse contact resistance between layers. Thus, when the concentration in a certain layer reaches saturation, the wax cannot penetrate the belt layer anymore.

In order to include saturation into the model, we derive the relation between concentration and inverse contact resistance at the interface between layers. One possibility is to assume that

$h_{i}=h_{0}\left(\mathrm{e}^{-\beta \frac{C_{\mathrm{b}}(t)}{C_{\mathrm{s}}}}-\mathrm{e}^{-\beta}\right)$,

where $h_{0}$ is a normalization, $C_{\mathrm{b}}$ is belt concentration, $C_{\mathrm{s}}$ is the saturation level of the belt, and $\beta$ is a constant. Another choice is

$h_{i}= \begin{cases}h_{0}\left(1-\frac{C_{\mathrm{b}}(t)}{C_{\mathrm{s}}}\right) & \text { for } C_{\mathrm{b}}<C_{\mathrm{s}} \\ 0 & \text { for } C_{\mathrm{b}}>C_{\mathrm{s}}\end{cases}$

Open Access This article is distributed under the terms of the Creative Commons Attribution Noncommercial License which permits any noncommercial use, distribution, and reproduction in any medium, provided the original author(s) and source are credited.

\section{References}

1. Wang EH, Liu WH (1988) Unsteady heat transfers through a multi-layer wall. Appl Energy 31(2):83-99

2. de Monte F (2002) An analytic approach to the unsteady heat conduction processes in one-dimensional composite media. Int $\mathbf{J}$ Heat Mass Transf 45(6):1333-1343

3. Pontrelli G, de Monte F (2007) Mass diffusion through two-layer porous media: an application to the drug-eluting stent. Int J Heat Mass Transf 50:3658-3669

4. Tamene Y, Bougriou C, Bessaïh R (2007) Thermal behaviour of a multilayer media in transient regime. Rev Energ Renouv 10(3):397-405

5. Hickson RI, Barry SI, Mercer GN (2009) Exact and numerical solutions for effective diffusivity and time lag through multiple layers. Anziam J 50:C682-C695

6. Bourouga B, Bardon JP (1998) Thermal contact resistance at the interface of double tubes assembled by plastic deformation. Exp Thermal Fluid Sci 18:168-181

7. Yeh CL, Wen CY (2001) An experimental investigation of thermal contact conductance across bolted joints. Exp Thermal Fluid Sci 25:349-357 
8. de Monte F (2000) Transient heat conduction in one-dimensional composite slab: a natural analytic approach. Int J Heat Mass Transf 43(19):3607-3619

9. Oturanç G, Sahin AZ (2001) Eigenvalue analysis of temperature distribution in composite walls. Int J Energy Res 25:1189-1196

10. Schuurmans AAP (1996) The conduction of heat in the fuse nip. Master Thesis. Technische Universiteit Eindhoven

11. Kopinga AJ, Kuipers J (2004) WaxDif: simulating wax diffusion inside a printer transportation belt, report of a project at Océ. Technische Universiteit Eindhoven

12. Zavinska O, Hlod A (2004) Model of wax diffusion and cleaning, report of a project at Océ. Technische Universiteit Eindhoven

13. Zapata PAM (2010) Modeling and computation of heat and moisture transport in paper. Master thesis, Technische Universiteit Eindhoven 